\title{
Myofacial pain dysfunction syndrome- management protocols revisited
}

\author{
Chaudhary A ${ }^{1}$, Dubey $\mathbf{P K}^{2}$, Patel $\mathbf{K}^{3}$, Patel $\mathbf{J R}^{4}$ \\ ${ }^{1}$ Dr Akashat Chaudhary, Reader, ${ }^{2}$ Dr Pavan Kumar Dubey, Reader, ${ }^{3}$ Dr Kavan Patel, Senior Lecturer, ${ }^{4}$ Jayanti R Patel, \\ Professor. All authors are affiliated with Department of Prosthodontics, NPDCH, Visnagar, Gujarat, India.
}

Address for Correspondence: Dr Pavan Kumar Dubey, Email: drpavan26@gmail.com

\begin{abstract}
Facial pain and its diagnosis have always posed a dilemma for the clinicians. The complex anatomy of the region compounded with the variability of symptoms involved, present a challenging situation during the management of orofacial pain. The dysfunction of these joint results in a large proportion of complaints presented to the dentists. With ever increasing refinements in diagnostic and treatment modalities, dramatic advances have been made in understanding the causes of facial pain related to joint and surrounding musculature. Still the mystery exists regarding the precise diagnosis and treatment of facial pain.
\end{abstract}

Keywords: Dentist, Mastication, Medical history taking, Fibromyalgia, Spasm, Temporomandibular joint disorders.

\section{Introduction}

Temporomandibular joint pain dysfunction syndrome is a term covering a variety of problems which include the entire scope of temporomandibular joint disorders originating either intra articular or extra articular [1].

Myofascial pain-dysfunction syndrome is a psycho physiologic disease that primarily involves the muscles of mastication. The condition is characterized by dull, aching, radiating pain that may become acute during use of the jaw, and mandibular dysfunction that generally involves a limitation of opening [2]. Frequently, myofascial pain is overlooked as a common cause of chronic pain because of frequent association with joint dysfunction and other pain disorders.

\section{Pathophysiology of MPD Syndrome}

As explained by Travell (1960) [1], the pathophysiology of this stress disorder of skeletal muscles may be outlined as follows: Noxious stimulation (due to such as mechanical, emotional, infectious, metabolic, nutritional, or a combination of these)

It has been observed that whenever the pain associated with skeletal muscle spasm is very severe, it is referred to a site from the muscle that is its source. The pain is referred from a small area of hypersensitivity located within the muscle or the fascia. These areas are termed as Trigger areas.

The response of muscles against the injury tends to gain momentum and results in a self representating cycle of spasmpain-spasm which limits the movements and result in fibrosis of tissues.

\section{Clinical Features}

Incidence: Women are affected by MPD syndrome more frequently than men, with the ratio ranging from 3:1 to 5:1[3]. The greatest incidence appears to be in the 20 to 40 years age group.

The patients suffering from MPD syndrome usually present with complaint of:

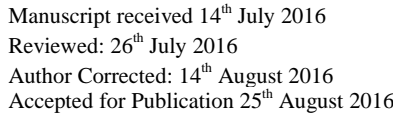


- Pain in a zone of reference (most important problem that causes patients to seek treatment).

- Trigger points in muscles which cause pain on stimulation.

- Taut muscle band.

- Limited jaw opening.

- Associated symptoms.

- Presence of contributing factors for onset of pain.

- No tenderness in temporomandibular joint.

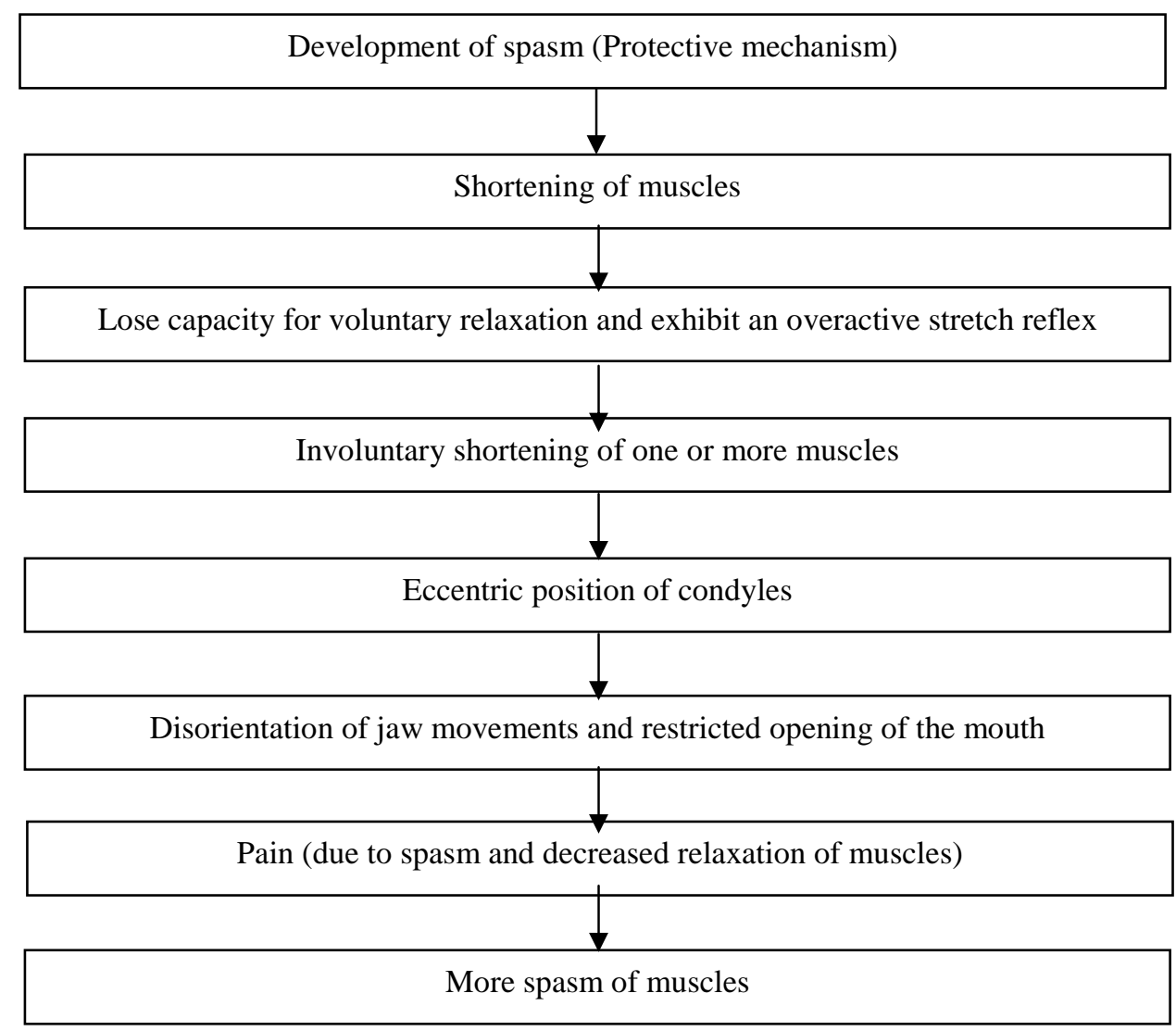

\section{Trigger points / Trigger zones / Trigger areas}

- Myofascial pain is characterized by pain referred from few hypersensitive areas termed as trigger areas / zones. A trigger point is defined as a localized tender area in taut band of skeletal muscle, tendon or ligaments.

- Points occur frequently in head, neck, shoulder, lower back.

- Any pressure on these areas may initiate pain referred to distant areas (called as zone of reference).

- Trigger areas develop due to direct / indirect trauma (parafunctional habits) to muscles, due to weakening of muscles (nutritional disturbances, lack of exercise, structural disharmony etc.).

- Trigger points range from $2.5 \mathrm{~mm}$ in diameter and may be active or latent.

- Palpating trigger points with deep finger pressure, elicits alteration in pain, in the zone of reference or causes radiation of pain towards the zone of reference.

- Patients behavioural reaction to firm palpation of trigger points is a distinguishing characteristic of myofascial pain and is termed a positive 'jump sign'. This reaction may include:

A) Withdrawal of head.

B) Wrinkling of face or forehead and desensation of skin.. 


\section{Management of MPD Syndrome}

I Diagnosis of the condition: The cardinal signs and symptoms of MPD syndrome are similar to those produced by many organic problems involving the temporomandibular joint and other non-articular conditions. Therefore, a careful history and thorough examination may be helpful in diagnosing the condition.

Radiographs may be helpful in diagnosing the condition if it has affected the bony structure also. X rays include: Transcranial, transpharyngeal, panoramic views, C.T. scans and MRI with arthroscopy can provide reliable diagnosis of the condition.

Arthrography can be useful in determining the position of meniscus (when internal derangement of temporomandibular joint is being considered).

\section{Certain lab tests are helpful}

\begin{tabular}{l|l}
$\begin{array}{l}\text { Complete blood cell count } \\
\text { Serum calcium, phosphorous and alkaline phosphatase } \\
\text { measurement. }\end{array}$ & $\begin{array}{l}\text { If infection is suspected. } \\
\text { For bone diseases. }\end{array}$ \\
$\begin{array}{l}\text { Serum uric acid determination. } \\
\text { Serum ceratinine and creatine phosphokinase levels. }\end{array}$ & $\begin{array}{l}\text { For gout. } \\
\text { Indicators of muscle disease. } \\
\text { ESR, Rheumatoid factor, Latex fixation test. }\end{array}$ \\
EMG & For rheumatoid arthritis. \\
Psychologic evaluation and psychometric testing. & For muscle function evaluation.
\end{tabular}

Treatment Approaches

The treatment of MPD syndrome should be geared towards complete management rather than symptomatic cure. Several treatment modalities have been recommended for MPD syndrome.

\section{Initial explanation of the problem}

The patients should be explained about the problem and its probable etiology. The psycho physiologic factor shouldn't be stressed while explaining the problem because patient may not accept it. Initial discussion should deal with muscle fatigue, spasm and explanation about the condition [2].

\section{Therapeutic modalities of treatment}

\section{a. Therapy at home}

- Intake of soft diet with small cut pieces. Jaw motion should be limited and wide opening should be avoided. Para functional habits such as clenching, grinding should be avoided (although patients are unaware of these habits, they should be instructed to check for clenching). Other habits such as fingernail biting, lip biting etc. should be avoided [4].

- Intermediate moist heat application for half an hour twice daily.

- Massage of the affected area using moderate kneading motion. This helps in return of venous blood, lymph and catabolites and reduces muscle pain and spasm.

\begin{tabular}{|l|l|}
\hline b. Short term medication \\
\hline Muscle relaxants and analgesics can be used. \\
\hline - Benzodiazepines 2mg-5mg QID (Diazepam) & - Aspirin 10gm TID \\
- Meprobomate 400mg TID & - Acetaminophen 650mg TID \\
Antidepressant drugs (for patients with long standing MPD & Propoxyphene 1-2 tabs TID. \\
syndrome and proven depression). Tricyclic antidepressants & \\
$25 \mathrm{mg}-75 \mathrm{mg}$ at bed time & \\
\hline
\end{tabular}




\section{c. Splint therapy}

$>$ If previously described forms of therapy are not successful or there is h/o tooth clenching etc., splint therapy should be considered [5].

$>$ According to Kawazoe5, 4 types of splints are used:
i. Stabilization splint.
ii. Relaxation splint.
iii. Resilient splint.
iv. Pivoting splint.

$>$ Hawley's type upper anterior splint is most effective because it prevents occlusion of posterior teeth and thereby prevents parafunctional activity. It is worn at night and 5-6 hours of day. Shouldn't be worn continuously as it results in supraeruption of posterior teeth. Platform of the splint should be flat.

\section{d. Physical therapy}

i) Ultrasound produces vibrations within the tissue that cause particle collision and release of energy. This results in production of heat and vibration which reduces muscle tension and increase in tissue elasticity. Moreover there is mild analgesic and anti-inflammatory action. Lasts for 10-15 minutes, given twice daily for 1-2 weeks[6].

ii) High voltage electrogalvanic stimulation involves the use of monophasic, pulsed direct current applied through an electrode placed on skin over the involved muscle.

- Activated at frequencies from 4-80 pulse per second, for 10-15 minutes, 2-3 times a week. Stimulation of muscle increases circulation, reduces pain and spasm and increases resistance to fatigue [7].

iii) Cryotherapy (cold therapy) [8]

$>$ Reduces tissue temperature, causes local analgesia and has anti-inflammatory effects and diminishes muscle spasm.

$>$ Cooling effect also creates vasoconstriction, reduces myoneural transmission and neuromuscular activity.

$>$ Vapour coolant spray (such as ethyl chloride or fluorimethane) is applied over the involved muscle by spraying for 10 seconds. Repeated for two more times with 10 seconds interval. Mandible is mobilized by gently stretching to maximum opening (also termed as spray and stretch activity).

$>$ Ice packs can be useful in acute phases of MPD syndrome. Cold application is used for 10-15 minutes; removed and reapplied after 5 to 10 minutes, 3-4 times daily.

e. Relaxation therapy: Because MPD syndrome is basically a problem related to increased muscle tension and spasm, any technique designed to induce muscle relaxation should be helpful. Among the modalities that have proven to be effective are biofeedback, conditioned relaxation and hypnosis [9]

i) Biofeedback

$>$ EMG biofeedback involves supplying the patient with visual or auditory information about the moment to moment contractile status of muscle being monitored. The patient then concentrates on relaxing the muscle and is reflected by reduction in level of graphic representation or audible sound.

$>$ The biofeedback is used for two 30-minutes sessions each week for 6 weeks.

Clarke and Kardachi (1977)[10] used biofeedback method in 7 patients suffering from MPD syndrome and achieved success by controlling parafunctional habits.

Dohrmann and Laskin (1978)[11] noted significant reduction in massetric EMG levels of patients treated with biofeedback.

ii) Conditioned relaxation :

Similar to biofeedback in its end results but differs in that the patients do not have the benefit of a feedback indicator.

iii) Other methods are hypnosis, transcendental meditation (TM) and yoga can also be useful in the treatment of MPD syndrome. 


\section{f. Anesthetic injections}

D Useful in extremely painful conditions. The injection of LA into tender and painful areas in muscle has been used for diagnostic and therapeutic purposes in patients with MPD syndrome [12].

$>0.5 \mathrm{cc}$ of LA that does not contain epinephrine or other vasoconstrictors are used.

\section{g. Transcutaneous Electrical Nerve Stimulation TENS[7]}

$>$ The use of TENS is based on the concept that stimulation of cutaneous branches of fifth nerve (trigeminal) creates an inhibitory effect on the trigeminal spinal tract nucleus, thus reducing the awareness of pain and relaxing the muscles.

$>$ Therapy lasts 30 minutes and should be repeated daily.

III. Final explanation of problem.

> When patients with MPD syndrome begin to show improvements of their symptoms and have gained confidence in doctors ability to deal with their problem, the relationship between stress and MPD syndrome should be discussed and explained [13].

IV. Psychologic Counseling

$>$ A group of patients are sometimes not able to identify and understand relationship between stress/strain and MPD syndrome and are unable to cope with stressful conditions [14]. Such patients should be referred to psychologists or psychiatrist for counseling. Psychological interventions are aimed at reducing high level of muscle tension or modifying the environment.

$>$ Treatment of contributing factors should be carried out.

\section{Summary}

The MPD syndrome is a psycho physiologically altered condition involving the muscles of mastication and cervical group of muscles. The condition characterized by dull aching, radiating pain often results in muscle spasm and restricted movements. An accurate diagnosis is accomplished by careful history taking and thorough examination. The application of proper therapy is related to the understanding that MPD syndrome is a stress induced psychophysiologic disease originating in muscles and not a temporomandibular joint disorder. Thus, the therapy should be directed towards reducing stress, rehabilitating the occlusion and relaxing the muscles to alleviate the condition.

Funding: Nil, Conflict of interest: None initiated, Permission from IRB: Yes

\section{References}

1. Mikhail M, Rosen H. History and etiology of myofascial pain-dysfunction syndrome. J Prosthet Dent 1980; 44(4): 438-44.

2. Laskin DM, Block S. Diagnosis and treatment of myofacial pain-dysfunction syndrome. J. Prosthet. Dent 1986; 56(1): 75-84.
3. Travell, J. Temporomandibular Joint Pain Referred From Muscles of the Head and Neck. J Prosthet. Dent. 1960; 10:745-763.

4. Dworkin SF. Benign chronic orofacial pain. Clinical criteria and therapeutic approaches. Postgrad Med 1983;74(3):239-42,245,247-8.

5. Kawazoe Y., Kotani H., Hamada T. and Yamada S. Effect of occlusal splints on the electromyographic activities of masseter muscles during maximum clenching in patients with myofacial pain-dysfunction syndrome. J Prosthet Dent 1980; 43(5):578-80.

6. Majlesi, J, Unalan H. High-power pain threshold ultrasound technique in the treatment of active myofascial triggers points: A randomized, double-blind, case - control study. Arch. Phys. Med. Rehabil 2004; 85(5): 833-6.

7. Graff-Radford, SB, Reeves JL, Baker RL, Chiu D. Effects of transcutaneous electrical nerve stimulation on myofascial pain and trigger point sensitivity. 1989; 37(1):1-5. 
8. Nadler SF, Weingand K, Kruser RJ. The physiologic basis and clinical applications of cryotherapy and thermotherapy for the pain practioner. Pain Physician. 2004; 7(3): 395-9.

9. Gary Elkins, Mark P. Jensen, David R. Patterson. Hypnotherapy for the Management of Chronic Pain.J Clin Exp Hypn. 2007; 55(3): 275-8.

10. Clarke NG, Kardachi BJ. The treatment of myofascial pain-dysfunction syndrome using the biofeedback principle. J. Periodont. 1977; 48 (10): 643-5.

11. Dohrmann RJ, Laskin DM. An evaluation of electromyographic biofeedback in the treatment of myofascial pain-dysfunction syndrome. J Am Dent
Assoc. 1978; 96(4): 656-62.

12. Fagiano G, Rolle G, Debernardi C, Catapano F, Pinnavaia M. Premedication in outpatient dental surgery under local. Minerva Stomatol. 1986; 35 (11):1027-31.

13. Gil IA, Barbosa CM, Pedro VM, Silverio KC, Goldfarb DP, Fusco V, Navarro CM. Multidisciplinary approach to chronic pain from myofascial pain dysfunction syndrome: a four-year experience at a Brazilian center. 1998 Jan; 16(1):17-25.

14. Kerstein RB. Disclusion time measurement studies: a comparison of disclusion time between chronic myofascial pain dysfunction patients and nonpatients: a population analysis. J Prosthet Dent 1994;72(5):473-80.

\section{How to cite this article?}

Chaudhary A, Dubey PK, Patel K, Patel JR. Myofacial pain dysfunction syndrome- management protocols revisited. Int J Med Res Rev 2016;4(8):1508-1513.doi:10.17511/ijmrr.2016.i08.35. 